\title{
Stability of Hyperbolic and Matter-Dominated Bounce Cosmologies From F(R,G) Modified Gravity at Late Evolution Stages
}

\author{
G. Navó, E. Elizalde \\ Institute of Space Sciences (IEEC-CSIC) Campus UAB, Carrer de Can Magrans, s/n 08193 Barcelona. \\ Recieved 5 December 2019. \\ Accepted 24 July 2020. \\ DOI:10.1142/S0219887820501625
}

\begin{abstract}
The stability of two different bounce scenarios from $F(R, \mathscr{G})$ modified gravity at later times is studied, namely a hyperbolic cosine bounce model and a matter-dominated one. After describing the main characteristics of $F(R, \mathscr{G})$ modified gravity, the two different bounce scenarios stemming from this theory are reconstructed and their stability at late stages is discussed. The stability of the hyperbolic cosine model is proven, while the concrete matter-bounce model here chosen does not seem to accomplish the necessary conditions to be stable at later times.
\end{abstract}

\section{Introduction}

The inflationary scenario (for an extensive review see [1] and references therein) provides the most popular scheme to answer the main problems of the original Big Bang cosmological model. It is able to explain (with the addition sometimes of quantum correction terms to the classical Einsteinian gravity), why the current observations converge on a flat, homogeneous and isotropic universe in accelerated expansion. This scenario is characterized by a huge expansion of the universe between the origin and the radiation era and provides the mechanism to generate nearly scale invariant primordial density fluctuations, which are thought to be the seeds of the large structures of our universe. Even though astronomical observations support such primordial fluctuations [2], they do not necessary imply inflation since there are several models in bouncing cosmologies that may also lead to them $[3,4]$.

The bounce scenario is actually the most usual alternative to inflation [3, 5]. One of its main features is the avoidance of the initial singularity, although there exist bounce models which do present it [6-8]. It is characterized by a contraction phase followed by the bounce point, which leads then to an expanding era. The inflection point where the Universe passes from one stage through to the other, the bounce point, occurs at time $t=t_{b}$. At this time, the scale factor $a\left(t_{b}\right) \equiv a_{b}$ and the Hubble parameter $H\left(t_{b}\right) \equiv H_{b}$ are

$$
\dot{a}_{b}=0, \ddot{a}_{b} \geq 0 ; H_{b}=\frac{\dot{a}_{b}}{a_{b}} .
$$

Thus, any Hubble rate involving a bounce scenario must fulfill these conditions.

In general, bouncing cosmologies emerge from the idea of avoiding the initial singularity in the scope of the Loop Quantum Cosmology matter bounce (see [9] and references therein), in scalar field theories, as for example in [10] or [11], and in modified gravities, which offer consistent descriptions of a bounce scenario (as an illustration one can consider [12, 13] or [14]). In the realms of General Relativity (GR) it is very difficult to handle 
the bouncing behavior since it leads to the violation of the Null Energy Condition (NEC) caused by the change in the sign of the Hubble rate at the bounce point [15]. An extended discussion on this issue is performed in [16] and [17]. In the present work, we focus on $F(R, \mathscr{G})$ modified gravity, which was introduced in [18-20] as a possible gravitational origin for Dark Energy. In the same vein, the literature provides numerous modified gravities [21] which have been explored in the scope of bouncing cosmologies such as $F(R)$ gravity [22], teleparallel gravity [23], modified Gauss-Bonnet gravity [24], or loop quantum gravity [25].

A well-known bounce scenario is the matter-dominated one, where the contraction is controlled by matter and, in addition, it is able to generate primordial density perturbations with a nearly scale invariant and adiabatic spectrum compatible with observations [26-28]. They are produced in the following way. Previous to the matter contraction, the fluctuations are inside the Hubble horizon, considered to be very large at that moment, what allows a vast region of the universe to be at causal contact. Once contraction has started, at some point perturbations become larger than the Hubble horizon, getting out of it and therefore getting frozen. After the bounce, the horizon grows, which makes the fluctuations to enter it again, allowing the production of the observed spectrum. However, this scenario presents some inconveniences to be addressed [29]. To mention one of the most important, it arises within the matter-dominated contraction epoch, which can be problematic leading to a growth of anisotropies, the so called BKL-instabilities [30]. In this work we will not focus on this aspect, conveniently discussed in the literature [31], where the problem is solved by adding an ekpyrotic contraction phase (for a review of the ekpyrotic model, see e.g. [32]).

Another popular bounce model is the hyperbolic cosine bounce. It is quite popular in the literature, since on top of being geodesically complete, it preserves causality and addresses the horizon problem [33-35]. Furthermore, working with this model one is able to provide relatively elegant solutions.

In this paper we study the stability at late time of a hyperbolic cosine bounce model and of a matter-dominated bounce scenario from an $F(R, \mathscr{G})$ gravity. We first provide a brief description of the $F(R, \mathscr{G})$ modified gravity theory, introducing the formalism and presenting some illustrative examples of the reconstructing method used. Then, we reconstruct both the hyperbolic and the matter-dominated models. Finally, we discuss the stability of the models at the later stages of the universe evolution.

\section{2. $\mathbf{F}(\mathbf{R}, \mathscr{G})$ gravity}

$F(R, \mathscr{G})$ gravity is a type of modified gravity that involves both the Ricci and the GaussBonnet scalars, which are higher-order corrections on the curvature tensor added to the Einstein's gravitational action. Its goal is to give an alternative way to explain the evolution of the universe as we currently understand it, by providing other possible options than dark energy and the initial singularity, both arising from the SCM. The presence of this term has also a mathematical basis and has been suggested as a feasible contribution by some string models.

In this section we present the general formalism of $F(R, \mathscr{G})$ modified gravity and work 
on an illustrative example for reconstructing it. We also discuss the necessary stability conditions for this type of gravity.

\subsection{Formalism}

First, we introduce the gravitational action for vacuum $F(R, \mathscr{G})$ gravity:

$$
\mathscr{S}=\frac{1}{2 \kappa} \int d^{4} x \sqrt{-g} F(R, \mathscr{G}),
$$

where $\mathscr{G}$ is the Gauss-Bonnet invariant, defined as

$$
\mathscr{G} \equiv R^{2}-4 R_{\alpha \beta} R^{\alpha \beta}+R_{\alpha \beta \rho \sigma} R^{\alpha \beta \rho \sigma},
$$

and $R$ is the Ricci scalar, which results from the contraction of the Ricci tensor, $R_{\alpha \beta}, R_{\alpha \beta \rho \sigma}$ being the Riemann tensor.

In order to obtain the gravitational equations of motion, we have to vary the gravitational action with respect to the metric tensor $g_{\mu \nu}$. Considering the flat FriedmannLemaitre-Robertson-Walker (FLRW) metric,

$$
d s^{2}=-d t^{2}+a^{2}(t)\left(d x^{2}+d y^{2}+d z^{2}\right),
$$

the gravitational equations become (see, for example, [36])

$$
\begin{array}{r}
2 \dot{H} F_{R}+8 H \dot{H} \dot{F}_{\mathscr{G}}=H \dot{F}_{R}-\ddot{F}_{R}+4 H^{3} \dot{F}_{\mathscr{G}}-4 H^{2} \ddot{F}_{\mathscr{G}}, \\
6 H^{2} F_{R}+24 H^{3} \dot{F}_{\mathscr{G}}=F_{R} R-F(R, \mathscr{G})-6 H \dot{F}_{R}+\mathscr{G} F_{\mathscr{G}},
\end{array}
$$

where $F_{\mathscr{G}} \equiv \frac{\partial F}{\partial \mathscr{G}} ; F_{R} \equiv \frac{\partial F}{\partial R}$, and $\mathscr{G}$ and $R$, in the FLRW metric, are equal to

$$
R=6\left(2 H^{2}+\dot{H}\right) ; \mathscr{G}=24 H^{2}\left(H^{2}+\dot{H}\right) \text {. }
$$

\subsection{Reconstructing $F(R, \mathscr{G})$ gravity}

In order to reconstruct the universe evolution under $F(R, \mathscr{G})$, only approximate solutions can be obtained analytically, since the equations of motion are highly complicated and one must do some approximation. Therefore, in order to obtain the cosmic evolution, we are led to use the reconstruction technique developed in [36]. We focus on a method which allows us to find the $F(R, \mathscr{G})$ function once the Hubble parameter is known.

Following the mentioned analysis, we express the Ricci and the Gauss-Bonet scalar of Eq. (6) in terms of the $e$-folding number, $N=\ln \left(a / a_{0}\right)$ :

$$
\begin{gathered}
R(N)=6\left(2 H^{2}(N)+H(N) H^{\prime}(N)\right), \\
\mathscr{G}(N)=24 H^{2}(N)\left(H^{2}(N)+H(N) H^{\prime}(N)\right),
\end{gathered}
$$

where the prime indicates derivative with respect to $N$.

Now, we assume a specific form for the functional of the Hubble parameter as, for example,

$$
H^{2}(N) \equiv P(N) .
$$


Then, from Eq. (7) and Eq. (8) we get

$$
R=12 P(N)+3 P^{\prime}(N) ; \quad \mathscr{G}=24 P^{2}(N)+12 P(N) P^{\prime}(N) .
$$

Therefore, the second Friedmann equation (Eq. (5)) can be written as

$$
\begin{aligned}
P(N) & \left(6 F_{R}+24 P(N) F_{\mathscr{G} G} \mathscr{G}^{\prime}(N)\right)-F_{R} R \\
& +F(R, \mathscr{G})+6 P(N) F_{R R} R^{\prime}(N)-\mathscr{G}(N) F_{\mathscr{G}}=0,
\end{aligned}
$$

which is a second order differential equation, which we can solve.

Hence, in order to obtain the solution for the $F(R, \mathscr{G})$ expression, we may first propose the Hubble rate we want to use, in order to relate it with $P(N), R$ and $\mathscr{G}$ and, finally, solve the differential equation.

As an illustrative example of the reconstruction method described, we now propose the following expression for the Hubble rate:

$$
H^{2}(N)=P(N)=P_{1} \exp \beta N,
$$

with $P_{1}>0$. Then, by substituting Eq. (12) into Eq. (10), we get

$$
\begin{aligned}
& N(\mathscr{G})=\frac{1}{2 \beta} \ln \frac{\mathscr{G}}{12 P_{1}^{2}(2+\beta)} \\
& P(\mathscr{G})=\frac{\mathscr{G}^{1 / 2}}{\left(12 P_{1}^{2}(2+\beta)\right)^{1 / 2}} .
\end{aligned}
$$

Moreover, in order to avoid very complicated equations, in what follows we will restrict to models with an $F(R, \mathscr{G})$ gravity expression of the form

$$
F(R, \mathscr{G})=R+f(\mathscr{G})
$$

Then, plugging Eq. (14) into Eq. (11) and considering Eq. (15), we find the differential equation

$$
\frac{2 \beta \mathscr{G}^{2}}{(2+\beta)^{2}}(3+2 \beta) F_{\mathscr{G} \mathscr{G}}+3^{1 / 2} \frac{\mathscr{G}^{1 / 2}}{(2+\beta)^{1 / 2}}-\mathscr{G}_{\mathscr{G}}+F(\mathscr{G})=0 .
$$

Quite often, the resulting differential equation may be very difficult to solve. If this happens, some leading order approximation will be needed. However, this is not the current case, where Eq. (16) can be solved in an analytic way, giving rise to

$$
F(\mathscr{G})=\frac{4 b}{a-2} \mathscr{G}^{1 / 2}+F_{0} \mathscr{G}^{1 / a}+F_{1} \mathscr{G},
$$

with $F_{0}$ and $F_{1}$ being integration constants and

$$
a=\frac{2 \beta(3+2 \beta)}{(2+\beta)^{2}} ; b=\frac{3^{1 / 2}}{(2+\beta)^{1 / 2}} .
$$

As another example, we focus on the case where the scale factor has exponential form, which fits with the characteristics of the bouncing cosmologies discussed in Sect. 1.

We consider the scale factor

$$
a(t)=e^{\alpha t^{2}},
$$


which has a Hubble rate of the form

$$
H(t)=2 \alpha t
$$

As has been mentioned previously, the first step is to rewrite the variables we work with in terms of $N$, namely

$$
H^{2}(N) \equiv P(N)=2 \alpha N
$$

Since we are interested in discussing the results at late evolution stages, we perform the limit when $N \rightarrow \infty$, getting $H^{\prime} \ll H$, what leads to a new expression for $\mathscr{G}(N)$, as

$$
\mathscr{G}(N) \simeq 96 \alpha^{2} N^{2} .
$$

Then, we obtain

$$
N(\mathscr{G})=\frac{\mathscr{G}^{1 / 2}}{4 \alpha \sqrt{6}}
$$

and, consequently

$$
P(\mathscr{G})=\frac{\mathscr{G}^{1 / 2}}{2 \sqrt{6}} .
$$

Furthermore, working with an $F(R, \mathscr{G})$ gravity of the type Eq. (15) allows to get the second Friedmann equation, Eq. (5), as

$$
24(P(N))^{2} \mathscr{G}^{\prime}(N) F_{\mathscr{G} \mathscr{G}}-\mathscr{G}(N) F_{\mathscr{G}}+6 P(N)=0 .
$$

At the end, we must solve the following non-trivial differential equation

$$
\frac{48 \alpha}{\sqrt{6}} \mathscr{G}^{3 / 2} F_{\mathscr{G} \mathscr{G}}-\mathscr{G}_{\mathscr{G}}+F(\mathscr{G})+\frac{\sqrt{6}}{2} \mathscr{G}^{1 / 2}=0 .
$$

Fortunately, in [36], after an exhaustive analysis, the following approximate solution for this second order differential equation was found

$$
F(\mathscr{G})=F_{1} G_{1}^{0} \frac{0}{1}\left(-\sqrt{\frac{\mathscr{G}}{6}} \mid 0,2\right),
$$

with $F_{1}$ being an integration constant. Thus, the final expression for the $F(R, \mathscr{G})$ function reads

$$
F(R, \mathscr{G})=R+F_{1} G_{1}^{0}{ }_{1}^{0}\left(-\sqrt{\frac{\mathscr{G}}{6}} \mid 0,2\right) .
$$

\subsection{Stability Conditions}

In this part, we introduce the necessary conditions for the background stability in the time evolution. 
Following the procedure in [37], we rewrite Eq. (11) in terms of the functional $P(N)$, only, as follows

$$
\begin{aligned}
24 & P^{2}(N)\left(48 P(N) P^{\prime}(N)+12 P(N) P^{\prime \prime}(N)\right. \\
& \left.+12 P^{\prime 2}(N)\right) F_{\mathscr{G} G}+6 P(N)\left(12 P^{\prime}(N)+3 P^{\prime \prime}(N)\right) F_{R R} \\
& -\left(24 P^{2}(N)+12 P(N) P^{\prime}(N)\right) F_{\mathscr{G}}-\left(6 P(N)+P^{\prime}(N)\right) F_{R} \\
& +F(R, \mathscr{G})=0 .
\end{aligned}
$$

Taking now into account Eq. (15) leads to $F_{R R}=0$ and $F_{R}=1$, and the previous expression becomes

$$
\begin{array}{r}
24 P^{2}(N)\left(48 P(N) P^{\prime}(N)+12 P(N) P^{\prime \prime}(N)+12 P^{\prime 2}(N)\right) F_{\mathscr{G} G} \\
-\left(24 P^{2}(N)+12 P(N) P^{\prime}(N)\right) F_{\mathscr{G}}+6 P(N)+F(\mathscr{G})=0,
\end{array}
$$

where the functional $P(N)$ is actually the background solution. Thus, since we are interested in finding the stability of this solution, we must perform the variation over its background $P(N)=P_{0}(N)+\delta P(N)$. In this way, we obtain

$$
\mathscr{J}_{1} \delta P^{\prime \prime}(N)+\mathscr{J}_{2} \delta, P^{\prime}(N)+\mathscr{J}_{3} \delta P(N)=0,
$$

with

$$
\begin{aligned}
& \mathscr{J}_{1} \equiv 288 P_{0}^{3}(N) F^{\prime \prime}\left(\mathscr{G}_{0}\right), \\
& \mathscr{J}_{2}= 432 P_{0}^{2}(N)\left(\left(2 P_{0}(N)+P_{0}^{\prime}(N)\right) F^{\prime \prime}\left(\mathscr{G}_{0}\right)\right. \\
&\left.+8 P_{0}(N)\left(\left(P_{0}^{\prime \prime}(N)\right)^{2}+P_{0}(N)\left(4 P_{0}^{\prime}(N)+P_{0}^{\prime \prime}(N)\right)\right)\right), \\
& \mathscr{J}_{3}= 6\left(1+24 P_{0}(N)\left(\left(-8 P_{0}^{2}(N)+3\left(P_{0}^{\prime}(N)\right)^{2}\right.\right.\right. \\
&+\left.P_{0}^{\prime \prime}(N)\right) F^{\prime \prime}\left(\mathscr{G}_{0}\right)+24 P_{0}(N)\left(4 P_{0}(N)+P_{0}^{\prime}(N)\right)\left(\left(P_{0}^{\prime}\right)^{2}\right. \\
&\left.\left.\left.+P_{0}(N)\left(4 P_{0}^{\prime}(N)+P_{0}^{\prime \prime}(N)\right)\right) F^{\prime \prime \prime}\left(\mathscr{G}_{0}\right)\right)\right) .
\end{aligned}
$$

Therefore, the stability conditions are $\frac{\mathscr{J}_{2}}{\mathscr{J}_{1}}>0$ and $\frac{\mathscr{I}_{3}}{\mathscr{J}_{1}}>0$.

\section{Bounce in $F(R, \mathscr{G})$ Gravity}

In this section, we reconstruct the $F(R, \mathscr{G})$ gravity for a hyperbolic and for a matterdominated bounce scenarios, and we discuss the stability of their cosmological solutions at late evolution stages.

\subsection{Hyperbolic Cosine Model}

We first focus on the hyperbolic cosine model, which is characterized by the following scale factor

$$
a(t)=\cosh (\lambda t),
$$

and, accordingly, by the corresponding Hubble parameter

$$
H(t)=\lambda \tanh (\lambda t) .
$$


In order to reconstruct the $F(R, \mathscr{G})$ gravity, we perform the same procedure as in Sect. 2.2. First, we obtain the e-folding number and the function $P(N)$

$$
H^{2}(N) \equiv P(N)=\lambda^{2}\left(1-e^{-2 N}\right) .
$$

Since we are interested in getting and discussing the solution at late time stages, we express $N(\mathscr{G})$ and $P(\mathscr{G})$ in the approximation $N \rightarrow \infty$, namely

$$
\begin{gathered}
N(\mathscr{G})=\ln \left(\frac{2 \lambda \sqrt{6}}{\sqrt{24 \lambda^{2}-\mathscr{G}}}\right), \\
P(\mathscr{G}) \simeq \lambda^{2} .
\end{gathered}
$$

If we now insert Eq. (38) and Eq. (39) into the Friedmann equation, Eq. (25), we get the differential equation to solve

$$
-\mathscr{G} F_{\mathscr{G} \mathscr{G}}+F(\mathscr{G})+\lambda^{2} \mathscr{G}=0,
$$

which leads to

$$
F(\mathscr{G})=\lambda^{2} \mathscr{G} \ln (\mathscr{G})+K \mathscr{G}
$$

being $K$ an integration constant.

Hence, our reconstructed $F(R, \mathscr{G})$ gravity which achieves Eq. (35) bounce behavior is

$$
F(R, \mathscr{G})=R+\lambda^{2} \mathscr{G} \ln (\mathscr{G})+K \mathscr{G} .
$$

Now we proceed to discuss the stability of this cosmological model at late stages. Taking into account that Eq. (37) at a late phase becomes $P(N) \simeq \lambda^{2}$, its derivatives are $P^{\prime}(N) \simeq 0$ and $P^{\prime \prime}(N) \simeq 0$. Consequently, we search for the expressions for the stability conditions by first obtaining $\mathscr{J}_{1}, \mathscr{J}_{2}$ and $\mathscr{J}_{3}$, as

$$
\begin{gathered}
\mathscr{J}_{1}=288 \lambda^{6} F^{\prime \prime}\left(\mathscr{G}_{0}\right), \\
\mathscr{J}_{2} \simeq 432 \lambda^{4}\left(12 \lambda^{6} F^{\prime \prime}\left(\mathscr{G}_{0}\right)=864 \lambda^{6} F^{\prime \prime}(N),\right. \\
\mathscr{J}_{3} \simeq 6\left(1-192 \lambda^{6} F^{\prime \prime}\left(\mathscr{G}_{0}\right)\right) .
\end{gathered}
$$

At this moment, we are able to discuss whether the stability conditions are satisfied in this model or not:

$$
\begin{gathered}
\frac{\mathscr{J}_{2}}{\mathscr{J}_{1}}=3>0, \\
\frac{\mathscr{J}_{3}}{\mathscr{J}_{1}}=\frac{1}{48 \lambda^{6} F^{\prime \prime}\left(\mathscr{G}_{0}\right)}-4>0
\end{gathered}
$$

As we can see, this hyperbolic model fulfills the stability conditions at late stages. 


\subsection{Matter-Dominated Bounce Model}

In this section we focus on a possible matter-bounce scenario [38], which is characterized by the following scale factor

$$
a(t)=a_{b}\left(1+q t^{2}\right)^{1 / 3}
$$

where $a_{b}$ is the scale factor at the bounce and $q$ is a constant. The corresponding Hubble parameter is

$$
H(t)=\frac{2 q t}{3\left(1+q t^{2}\right)} .
$$

Following the same reconstruction method as in the previous sections, we first obtain $P(N)$

$$
H^{2}(N) \equiv P(N)=\frac{4}{3} e^{-3 N},
$$

where $N \simeq \frac{2}{3} \ln (t)$. Then, putting Eq. (50) into Eq. (10), we get the number of e-folds in terms of the Gauss-Bonnet scalar, $N(\mathscr{G})$, and also $P(\mathscr{G})$, as

$$
\begin{gathered}
N(\mathscr{G})=\frac{1}{6} \ln \left(-\frac{64}{27 \mathscr{G}}\right), \\
P(\mathscr{G})=\frac{\sqrt{-27 \mathscr{G}}}{18} .
\end{gathered}
$$

Substituting Eq. (52) and Eq. (51) into the Friedmann equation, we are able to solve the differential equation, with the result

$$
f(\mathscr{G})=C_{1} \mathscr{G}^{1 / 12}+C_{2} \mathscr{G}+\frac{2 \sqrt{3}}{5} \sqrt{-\mathscr{G}},
$$

and, therefore

$$
F(R, \mathscr{G})=R+C_{1} \mathscr{G}^{1 / 12}+C_{2} \mathscr{G}+\frac{2 \sqrt{3}}{5} \sqrt{-\mathscr{G}} .
$$

As in the previous section, we are interested in discussing if this particular matterbounce scenario fulfills the stability conditions. Taking into account Eq. (50), the derivatives of $P(N)$ are

$$
\begin{gathered}
P^{\prime}(N)=-\frac{4}{3} e^{-3 N} \\
P^{\prime \prime}(N)=4 e^{-3 N}
\end{gathered}
$$

Therefore, considering the same leading order approximation at late stages, $N \rightarrow \infty$, we obtain the expressions for $\mathscr{J}_{1}, \mathscr{J}_{2}$ and $\mathscr{J}_{3}$ :

$$
\mathscr{J}_{1}=\frac{1048}{81} e^{-9 N} F^{\prime \prime}\left(\mathscr{G}_{0}\right)
$$




$$
\begin{aligned}
\mathscr{J}_{2} & =\frac{256}{3} e^{-6 N}\left(-\frac{4}{9} e^{-3 N} F^{\prime \prime}\left(\mathscr{G}_{0}\right)+\frac{13312}{243} e^{-9 N}\right) \\
& \simeq-\frac{1024}{27} e^{-9 N} F^{\prime \prime}\left(\mathscr{G}_{0}\right)
\end{aligned}
$$

and

$$
\mathscr{J}_{3} \simeq 6
$$

Finally, we arrive the following expressions

$$
\begin{gathered}
\frac{\mathscr{J}_{2}}{\mathscr{J}_{1}}=-\frac{2}{3}<0, \\
\frac{\mathscr{J}_{3}}{\mathscr{J}_{1}}=\frac{486}{2048} \frac{e^{9 N}}{F^{\prime \prime}\left(\mathscr{G}_{0}\right)}=\infty>0 .
\end{gathered}
$$

As we can observe from these relations, this specific scenario does not accomplish the background stability conditions at late time evolution.

\section{Conclusions}

In this paper, after discussing the main characteristics of $F(R, \mathscr{G})$ modified gravity, we have presented in detail the reconstruction method used in the rest of the paper and considered some illustrative examples, in particular the exponential bounce model. We have also described the conditions needed for a cosmological model evolution to be stable and subsequently applied the reconstruction method for the hyperbolic cosine bounce model, in which case we have successfully found a possible $F(R, \mathscr{G})$ expression which achieves the goal, and we have then proven its stability at late cosmological times (for interesting alternative methods, see [39-41]). That this is rather non-trivial is proven by the second example, where we have carried out the same analysis in a matter-bounce cosmology, which is actually consistent with astronomical observations of the early universe, as discussed in Sect. 1. Actually, we have proven that, at a later epoch, $N \rightarrow \infty$, this second specific model does not fulfill the stability conditions and, therefore, it is non-viable. This different stability behavior could be explained by the different evolution of the Hubble radius in both cases and, therefore, a different generation era of the perturbation modes. In the cosine hyperbolic case it goes to zero asymptotically and therefore the perturbation modes generate near the bounce. On the other hand, in the matter-dominated bounce, the Hubble radius diverges asymptotically and thus the perturbation modes generate deeply in the contracting regime far away from the bounce, suggesting that it may be inter-related with the results obtained in Sect. 3. In order to recover the stability in the matter bounce model, one could use the lagrange multyplier $F(R, \mathscr{G})$ model, which seems to make the matter bounce stable [42].

\section{Acknowledgements}

E.E. has been partly supported by MINECO (Spain), project FIS2016-76363-P, and by AGAUR (Catalan Government), project 2017-SGR-247. We thank Tanmoy Paul for interesting comments. 


\section{References}

[1] J. Alberto Vázquez, Luis E. Padilla, and Tonatiuh Matos. Inflationary Cosmology: From Theory to Observations. arXiv e-prints, art. arXiv:1810.09934, Oct 2018.

[2] Planck Collaboration, P. A. R. Ade, N. Aghanim, M. Arnaud, F. Arroja, M. Ashdown, J. Aumont, C. Baccigalupi, M. Ballardini, A. J. Banday, R. B. Barreiro, N. Bartolo, E. Battaner, K. Benabed, A. Benoît, A. Benoit-Lévy, J. P. Bernard, M. Bersanelli, P. Bielewicz, J. J. Bock, A. Bonaldi, L. Bonavera, J. R. Bond, J. Borrill, F. R. Bouchet, F. Boulanger, M. Bucher, C. Burigana, R. C. Butler, E. Calabrese, J. F. Cardoso, A. Catalano, A. Challinor, A. Chamballu, R. R. Chary, H. C. Chiang, P. R. Christensen, S. Church, D. L. Clements, S. Colombi, L. P. L. Colombo, C. Combet, D. Contreras, F. Couchot, A. Coulais, B. P. Crill, A. Curto, F. Cuttaia, L. Danese, R. D. Davies, R. J. Davis, P. de Bernardis, A. de Rosa, G. de Zotti, J. Delabrouille, F. X. Désert, J. M. Diego, H. Dole, S. Donzelli, O. Doré, M. Douspis, A. Ducout, X. Dupac, G. Efstathiou, F. Elsner, T. A. Enßlin, H. K. Eriksen, J. Fergusson, F. Finelli, O. Forni, M. Frailis, A. A. Fraisse, E. Franceschi, A. Frejsel, A. Frolov, S. Galeotta, S. Galli, K. Ganga, C. Gauthier, M. Giard, Y. Giraud-Héraud, E. Gjerløw, J. González-Nuevo, K. M. Górski, S. Gratton, A. Gregorio, A. Gruppuso, J. E. Gudmundsson, J. Hamann, W. Handley, F. K. Hansen, D. Hanson, D. L. Harrison, S. Henrot-Versillé, C. Hernández-Monteagudo, D. Herranz, S. R. Hildebrand t, E. Hivon, M. Hobson, W. A. Holmes, A. Hornstrup, W. Hovest, Z. Huang, K. M. Huffenberger, G. Hurier, A. H. Jaffe, T. R. Jaffe, W. C. Jones, M. Juvela, E. Keihänen, R. Keskitalo, J. Kim, T. S. Kisner, R. Kneissl, J. Knoche, M. Kunz, H. Kurki-Suonio, G. Lagache, A. Lähteenmäki, J. M. Lamarre, A. Lasenby, M. Lattanzi, C. R. Lawrence, R. Leonardi, J. Lesgourgues, F. Levrier, A. Lewis, M. Liguori, P. B. Lilje, M. Linden-Vørnle, M. López-Caniego, P. M. Lubin, Y. Z. Ma, J. F. Macías-Pérez, G. Maggio, D. Maino, N. Mandolesi, A. Mangilli, M. Maris, P. G. Martin, E. Martínez-González, S. Masi, S. Matarrese, P. McGehee, P. R. Meinhold, A. Melchiorri, L. Mendes, A. Mennella, M. Migliaccio, S. Mitra, M. A. MivilleDeschênes, D. Molinari, A. Moneti, L. Montier, G. Morgante, D. Mortlock, A. Moss, M. Münchmeyer, D. Munshi, J. A. Murphy, P. Naselsky, F. Nati, P. Natoli, C. B. Netterfield, H. U. Nørgaard-Nielsen, F. Noviello, D. Novikov, I. Novikov, C. A. Oxborrow, F. Paci, L. Pagano, F. Pajot, R. Paladini, S. Pandolfi, D. Paoletti, F. Pasian, G. Patanchon, T. J. Pearson, H. V. Peiris, O. Perdereau, L. Perotto, F. Perrotta, V. Pettorino, F. Piacentini, M. Piat, E. Pierpaoli, D. Pietrobon, S. Plaszczynski, E. Pointecouteau, G. Polenta, L. Popa, G. W. Pratt, G. Prézeau, S. Prunet, J. L. Puget, J. P. Rachen, W. T. Reach, R. Rebolo, M. Reinecke, M. Remazeilles, C. Renault, A. Renzi, I. Ristorcelli, G. Rocha, C. Rosset, M. Rossetti, G. Roudier, M. Rowan-Robinson, J. A. Rubiño-Martín, B. Rusholme, M. Sandri, D. Santos, M. Savelainen, G. Savini, D. Scott, M. D. Seiffert, E. P. S. Shellard, M. Shiraishi, L. D. Spencer, V. Stolyarov, R. Stompor, R. Sudiwala, R. Sunyaev, D. Sutton, A. S. Suur-Uski, J. F. Sygnet, J. A. Tauber, L. Terenzi, L. Toffolatti, M. Tomasi, M. Tristram, T. Trombetti, M. Tucci, J. Tuovinen, L. Valenziano, J. Valiviita, B. Van Tent, P. Vielva, F. Villa, L. A. Wade, 
B. D. Wandelt, I. K. Wehus, M. White, D. Yvon, A. Zacchei, J. P. Zibin, and A. Zonca. Planck 2015 results. XX. Constraints on inflation. Astronomy and Astrophysics, 594: A20, Sep 2016. doi: 10.1051/0004-6361/201525898.

[3] Robert H. Brandenberger. The Matter Bounce Alternative to Inflationary Cosmology. arXiv e-prints, art. arXiv:1206.4196, Jun 2012.

[4] S. D. Odintsov and V. K. Oikonomou. Matter bounce loop quantum cosmology from F (R ) gravity. Physical Review D, 90(12):124083, Dec 2014. doi: 10.1103/PhysRevD.90.124083.

[5] Robert H. Brandenberger. Alternatives to the Inflationary Paradigm of Structure Formation. In International Journal of Modern Physics Conference Series, volume 1 of International Journal of Modern Physics Conference Series, pages 67-79, Jan 2011. doi: 10.1142/S2010194511000109.

[6] S. D. Odintsov and V. K. Oikonomou. Big-Bounce with Finite-time Singularity: The $F(R)$ Gravity Description. arXiv e-prints, art. arXiv:1512.04787, Dec 2015.

[7] S. D. Odintsov and V. K. Oikonomou. Bouncing cosmology with future singularity from modified gravity. Physical Review D, 92(2):024016, Jul 2015. doi: 10.1103/ PhysRevD.92.024016.

[8] Kazuharu Bamba, Shin'ichi Nojiri, and Sergei D. Odintsov. The future of the universe in modified gravitational theories: approaching a finite-time future singularity. Journal of Cosmology and Astro-Particle Physics, 2008(10):045, Oct 2008. doi: 10.1088/1475-7516/2008/10/045.

[9] Abhay Ashtekar and Parampreet Singh. Loop quantum cosmology: a status report. Classical and Quantum Gravity, 28(21):213001, Nov 2011. doi: 10.1088/0264-9381/ 28/21/213001.

[10] Yi-Fu Cai, Robert Brandenberger, and Xinmin Zhang. The matter bounce curvaton scenario. Journal of Cosmology and Astro-Particle Physics, 2011(3):003, Mar 2011. doi: 10.1088/1475-7516/2011/03/003.

[11] Hiroki Matsui, Fuminobu Takahashi, and Takahiro Terada. Bouncing Universe from Nothing. arXiv e-prints, art. arXiv:1904.12312, Apr 2019.

[12] S. D. Odintsov, V. K. Oikonomou, and Emmanuel N. Saridakis. Superbounce and loop quantum ekpyrotic cosmologies from modified gravity: $F(R), F(G)$ and $F(T)$ theories. Annals of Physics, 363:141-163, Dec 2015. doi: 10.1016/j.aop.2015.08.021.

[13] V. K. Oikonomou. Superbounce and loop quantum cosmology ekpyrosis from modified gravity. Astrophysics and Space Science, 359:30, Sep 2015. doi: 10.1007/ s10509-015-2478-1.

[14] S. D. Odintsov and V. K. Oikonomou. $\Lambda$ CDM bounce cosmology without $\Lambda$ CDM : The case of modified gravity. Physical Review D, 91(6):064036, Mar 2015. doi: 10.1103/PhysRevD.91.064036.

[15] Diana Battefeld and Patrick Peter. A critical review of classical bouncing cosmologies. Physics Reports, 571:1-66, Apr 2015. doi: 10.1016/j.physrep.2014.12.004.

[16] Kurt Hinterbichler, Austin Joyce, Justin Khoury, and Godfrey E. J. Miller. DiracBorn-Infeld Genesis: An Improved Violation of the Null Energy Condition. Physical Review Letters, 110(24):241303, Jun 2013. doi: 10.1103/PhysRevLett.110.241303. 
[17] Benjamin Elder, Austin Joyce, and Justin Khoury. From satisfying to violating the null energy condition. Physical Review D, 89(4):044027, Feb 2014. doi: 10.1103/ PhysRevD.89.044027.

[18] Shin'ichi Nojiri and Sergei D. Odintsov. Modified Gauss Bonnet theory as gravitational alternative for dark energy. Physics Letters B, 631(1-2):1-6, Dec 2005. doi: 10.1016/j.physletb.2005.10.010.

[19] Guido Cognola, Emilio Elizalde, Shin'Ichi Nojiri, Sergei D. Odintsov, and Sergio Zerbini. Dark energy in modified Gauss-Bonnet gravity: Late-time acceleration and the hierarchy problem. Physical Review D, 73(8):084007, Apr 2006. doi: 10.1103/ PhysRevD.73.084007.

[20] Mariafelicia De Laurentis, Mariacristina Paolella, and Salvatore Capozziello. Cosmological inflation in $f(r, \mathscr{G})$ gravity. Phys. Rev. D, 91:083531, Apr 2015. doi: 10.1103/ PhysRevD.91.083531. URL https://link.aps.org/doi/10.1103/PhysRevD. 91.083531.

[21] S. Nojiri, S. D. Odintsov, and V. K. Oikonomou. Modified gravity theories on a nutshell: Inflation, bounce and late-time evolution. Physics Reports, 692:1-104, Jun 2017. doi: 10.1016/j.physrep.2017.06.001.

[22] Carlos Barragán, Gonzalo J. Olmo, and Hèlios Sanchis-Alepuz. Bouncing cosmologies in Palatini $\mathrm{f}(\mathrm{R})$ gravity. Physical Review D, 80(2):024016, Jul 2009. doi: 10.1103/PhysRevD.80.024016.

[23] Yi-Fu Cai, Shih-Hung Chen, James B. Dent, Sourish Dutta, and Emmanuel N. Saridakis. Matter bounce cosmology with the $\mathrm{f}(\mathrm{T})$ gravity. Classical and Quantum Gravity, 28(21):215011, Nov 2011. doi: 10.1088/0264-9381/28/21/215011.

[24] Kazuharu Bamba, Andrey N. Makarenko, Alexandr N. Myagky, and Sergei D. Odintsov. Bouncing cosmology in modified Gauss-Bonnet gravity. Physics Letters B, 732:349-355, May 2014. doi: 10.1016/j.physletb.2014.04.004.

[25] Gonzalo J. Olmo and Parampreet Singh. Covariant effective action for loop quantum cosmology à la Palatini. Journal of Cosmology and Astro-Particle Physics, 2009(1): 030, Jan 2009. doi: 10.1088/1475-7516/2009/01/030.

[26] M. Novello and S. E. Perez Bergliaffa. Bouncing cosmologies. Physics Reports, 463 (4):127-213, Jul 2008. doi: 10.1016/j.physrep.2008.04.006.

[27] Emilio Elizalde, Jaume Haro, and Sergei D. Odintsov. Quasimatter domination parameters in bouncing cosmologies. Physical Review D, 91(6):063522, Mar 2015. doi: 10.1103/PhysRevD.91.063522.

[28] Jaume de Haro and Yi-Fu Cai. An extended matter bounce scenario: current status and challenges. General Relativity and Gravitation, 47:95, Aug 2015. doi: 10.1007/ s10714-015-1936-y.

[29] Robert Brandenberger and Patrick Peter. Bouncing Cosmologies: Progress and Problems. Foundations of Physics, 47(6):797-850, Jun 2017. doi: 10.1007/ s10701-016-0057-0.

[30] V. A. Belinsky, I. M. Khalatnikov, and E. M. Lifshitz. Oscillatory approach to a singular point in the relativistic cosmology. Adv. Phys., 19:525-573, 1970. doi: 10.1080/00018737000101171. 
[31] Yi-Fu Cai, Robert Brandenberger, and Patrick Peter. Anisotropy in a non-singular bounce. Classical and Quantum Gravity, 30(7):075019, Apr 2013. doi: 10.1088/ 0264-9381/30/7/075019.

[32] Justin Khoury, Burt A. Ovrut, Paul J. Steinhardt, and Neil Turok. Ekpyrotic universe: Colliding branes and the origin of the hot big bang. Physical Review D, 64(12): 123522, Dec 2001. doi: 10.1103/PhysRevD.64.123522.

[33] Tirthabir Biswas, Anupam Mazumdar, and Warren Siegel. Bouncing universes in string-inspired gravity. Journal of Cosmology and Astrophysics, 2006(3):009, Mar 2006. doi: 10.1088/1475-7516/2006/03/009.

[34] Tirthabir Biswas, Alexey S. Koshelev, Anupam Mazumdar, and Sergey Yu. Vernov. Stable bounce and inflation in non-local higher derivative cosmology. Journal of Cosmology and Astrophysics, 2012(8):024, Aug 2012. doi: 10.1088/1475-7516/2012/08/ 024.

[35] Tirthabir Biswas and Anupam Mazumdar. Super-inflation, non-singular bounce, and low multipoles. Classical and Quantum Gravity, 31(2):025019, dec 2013. doi: 10. 1088/0264-9381/31/2/025019. URL https://doi .org/10.1088\%2F0264-9381\% $2 \mathrm{~F} 31 \% 2 \mathrm{~F} 2 \% 2 \mathrm{~F} 025019$.

[36] S. D. Odintsov, V. K. Oikonomou, and S. Banerjee. Dynamics of inflation and dark energy from F (R , G) gravity. Nuclear Physics B, 938:935-956, Jan 2019. doi: 10.1016/j.nuclphysb.2018.07.013.

[37] Anna Escofet and Emilio Elizalde. Gauss-Bonnet modified gravity models with bouncing behavior. Modern Physics Letters A, 31(17):1650108, Jun 2016. doi: 10.1142/S021773231650108X.

[38] Emmanuel N. Saridakis, Shreya Banerjee, and R. Myrzakulov. Bounce and cyclic cosmology in new gravitational scalar-tensor theories. Physical Review D, 98(6): 063513, Sep 2018. doi: 10.1103/PhysRevD.98.063513.

[39] Salvatore Capozziello, Mariafelicia de Laurentis, and Sergei D. Odintsov. Noether symmetry approach in Gauss-Bonnet cosmology. Modern Physics Letters A, 29(30): 1450164-204, September 2014. doi: 10.1142/S0217732314501648.

[40] Salvatore Capozziello, Andrey N. Makarenko, and Sergei D. Odintsov. Gauss-Bonnet dark energy by Lagrange multipliers. Physical Review D, 87(8):084037, April 2013. doi: 10.1103/PhysRevD.87.084037.

[41] Salvatore Capozziello, Emilio Elizalde, Shin'ichi Nojiri, and Sergei D. Odintsov. Accelerating cosmologies from non-local higher-derivative gravity. Physics Letters B, 671(1):193-198, January 2009. doi: 10.1016/j.physletb.2008.11.060.

[42] E. Elizalde, S. D. Odintsov, V. K. Oikonomou, and Tanmoy Paul. Extended matter bounce scenario in ghost free $\mathrm{f}(\mathrm{R}, \mathrm{G})$ gravity compatible with GW170817. Nuclear Physics B, 954:114984, May 2020. doi: 10.1016/j.nuclphysb.2020.114984. 